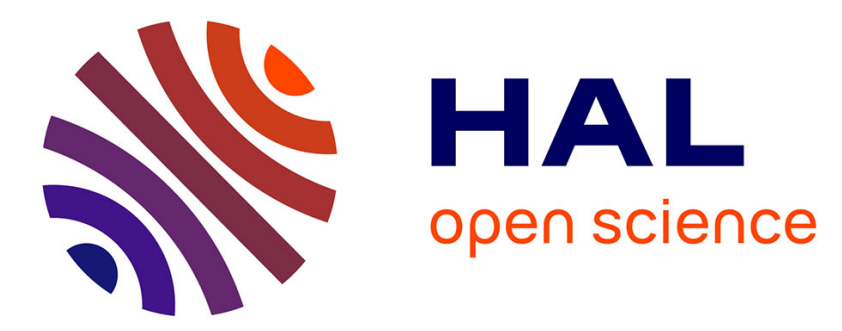

\title{
Modeling the Partial Renewable Power Curtailment for Transmission Network Management
}

\author{
Alessio Iovine, Duc-Trung Hoang, Sorin Olaru, Jean Maeght, Patrick
} Panciatici, Manuel Ruiz

\section{- To cite this version:}

Alessio Iovine, Duc-Trung Hoang, Sorin Olaru, Jean Maeght, Patrick Panciatici, et al.. Modeling the Partial Renewable Power Curtailment for Transmission Network Management. 2021 IEEE PowerTech Conference, Jun 2021, Madrid, Spain. 10.1109/PowerTech46648.2021.9494993 • hal-03004441v2

\section{HAL Id: hal-03004441 \\ https://hal-centralesupelec.archives-ouvertes.fr/hal-03004441v2}

Submitted on 29 Nov 2020 (v2), last revised 27 Oct 2021 (v3)

HAL is a multi-disciplinary open access archive for the deposit and dissemination of scientific research documents, whether they are published or not. The documents may come from teaching and research institutions in France or abroad, or from public or private research centers.
L'archive ouverte pluridisciplinaire HAL, est destinée au dépôt et à la diffusion de documents scientifiques de niveau recherche, publiés ou non, émanant des établissements d'enseignement et de recherche français ou étrangers, des laboratoires publics ou privés. 


\section{Modeling the Partial Renewable Power Curtailment for Transmission Network Management}

\author{
Alessio Iovine, Duc-Trung Hoang, Sorin Olaru \\ Laboratory of Signals and Systems (L2S) \\ CentraleSupélec, Paris-Saclay University \\ Gif-sur-Yvette, France \\ \{alessio.iovine, trung.hoang-duc, sorin.olaru\}@ centralesupelec.fr
}

\author{
Jean Maeght, Patrick Panciatici, \\ Manuel Ruiz \\ French Transmission System Operator \\ Réseau de Transport d'Électricité (RTE) \\ Paris, France \\ \{name.surename\}@rte-france.com
}

\begin{abstract}
Congestion problems are increasing in number in power transmission networks due to the increment of renewable power sources along it. To reduce their impact, Transmission System Operators (TSOs) as the French RTE use network reconfiguration or renewable power curtailment in complex subtransmission areas. The operators need enhanced methodological tools to better address the optimal power flow management problem by also using novel levers as for example the storage devices. This paper proposes mathematical models which integrate the possibility to partially curtail the renewable power in the form of a dynamical system representing the transmission network, also considering storage devices.
\end{abstract}

Index Terms-Modeling, transmission network, partial curtailment, energy storage.

\section{INTRODUCTION}

An increasing number of transmission networks are or will be congested in the near future due to the increase of renewable generation. For example, in France, wind turbines are usually built in rural areas, where the electrical grid is less developed [1]. Consequently, wind turbines aggregation may require the addition of new lines to enable produced electricity delivery. To limit this necessity and the extra costs it requires, new congestion management methods implementing advanced constraints handling techniques are nowadays investigated [2], [3], [4], [5], [6]. These new congestion management methods are model-based, and allow for a better utilisation of the available resources, in terms of maximizing the renewable power production and reduction of impact on the transmission lines. They consider the possible power curtailment for renewables and use storage devices in online optimization strategies by including power lines constraints, control action delays, and uncertainties due to power generation and model approximations.

Interest is raising on the possibility to split the whole transmission network in several subtransmission areas, and to optimally manage them via Model Predictive Control (MPC) [7]. In the current literature, due to the unique possibility to have on/off decisions for power curtailment, the existing models only consider generation ramping and no information is kept about the amount of real-time generated power. For example, the works in [2] and [3] describe a dynamical model based on Power Transfer Distribution Factor (PTDF) (see [8, 9]) that allows only on/off curtailment decisions, and consequently eludes the manipulation of elements on power generation. As the possibility to consider partial curtailment can now be envisaged by the Transmission System Operators (TSOs), the present paper introduces a dynamical model describing the whole set of elements that are needed to partially curtail the renewable power. The proposed approach is based on PTDF modelling framework, and targets the possibility to use model-based optimisation techniques to optimally manage a subtransmission area congestion situation via renewable power partial curtailment and storage devices.

The target of the present paper is to provide a dynamical model that dependably describes the system functioning and is suitable for model-based optimal management of a zone. Due to communication constraints, only a local description of the zone is available and the connection with the remaining network is defined as a perturbation acting on the zone. The main challenge in controlling an isolated zone is to operate local control actions with respect to the global power flow taking place at the boundaries of the zone. Indeed, due to security and practical reasons, it is not possible to take decision based on the state measurement at the scale of the whole network. Consequently, to obtain a close-to-reality dynamical model for the considered zone is a challenging problem. Moreover, we propose a control-oriented modeling approach.

The ultimate goal of the present paper is to validate a model capable to consider the possibility for transmission networks to partially curtail power from renewables and to use storage devices for online optimization strategies that consider power lines constraints, control action delays, and uncertainties due to power generation and model approximations.

The paper is organized as follows. Section III introduces the considered modeling. Simulations validating the linearized dynamics are carried out in Section III] while concluding remarks are outlined in Section IV.

Notations:

- $\mathcal{Z}^{N}$ is the set of nodes in the considered zone; $n^{N}$ is its cardinality. $P_{n}^{T}$ is the power generated in the transmission network outside the considered zone that impacts on it.

- $\mathcal{Z}^{C} \subset \mathcal{Z}^{N}$ is the set of nodes where the curtailment of the generated power is allowed; $n^{C}$ is its cardinality. $P_{n}^{G}$ is the generated power, while $P_{n}^{C}$ is the curtailed one at node $n \in \mathcal{Z}^{C} . P_{n}^{A}$ is the available renewable power that 


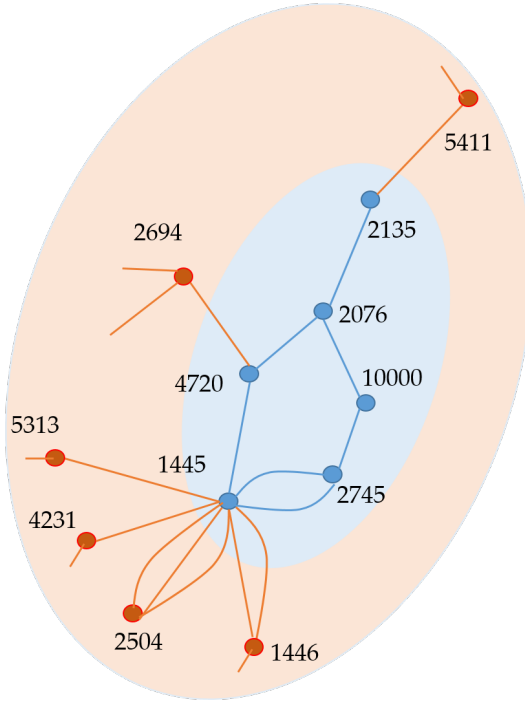

Fig. 1. The considered zone (blue nodes) and its connection to the entire power network (red nodes). Each node is described by its number. The considered dynamical system describes the dynamics of the blue nodes and the branches among them. The power flow interaction among the blue nodes and the magenta nodes is described as an uncontrolled generated/absorbed power.

can be generated each sampling time. Also, we define $P_{n}^{P}$ as the really produced renewable power.

- $\mathcal{Z}^{B} \subset \mathcal{Z}^{N}$ is the set of nodes with a battery; $n^{B}$ is its cardinality. $P_{n}^{B}$ is the power injected from the battery on node $n \in \mathcal{Z}^{C}$, while $E_{n}^{B}$ describes the battery energy at the same node.

- $\mathcal{Z}^{L} \subset\left\{(i, j) \in\left\{1, \ldots, n^{N}\right\} \times\left\{1, \ldots, n^{N}\right\}\right\}$ is the set of power lines in the considered zone; $n^{L}$ is its cardinality. $F_{i j}$ represents the power flow on the line $i j$.

We use the operator diag to describe a diagonal matrix composed by the considered elements. The operator col produces a single column vector composed by the aggregation of other vectors. That is, given $m$ vectors $s_{i} \in \mathbb{R}^{n}, i=1, \ldots, m$, the resulting vector $s=\operatorname{col}\left[s_{i}\right], i=1,2, \ldots, m$, will be:

$$
s=\operatorname{col}\left[s_{i}\right]=\left[\begin{array}{llll}
s_{1}^{T} & s_{2}^{T} & \ldots & s_{m}^{T}
\end{array}\right]^{T} \in \mathbb{R}^{n m} .
$$

\section{MODELING}

The models introduced in this Section describe a situation where partial curtailment is allowed. Both the power that could be produced and the one that is actually delivered are taken into account.

Approximate linear models resulting in a Direct Current (DC) description of the Alternate Current (AC) nonlinear ones are usually considered to operate model-based control of subtransmission areas named zones. Variations in power flows are represented by a linearization, where the matrices computation is based on PTDF (see [8, 9]). PTDF models are commonly used in power systems network modeling and analysis; a description is given in [2, 8, 9]. We use the realistic tool MATPOWER (see [10]) with the full French grid data set to simulate the power transmission line under various scenarios and to calculate the parameters for the matrices. For the exemplification, a subtransmission area (zone) is depicted in Figure 1, geographically close to Dijon, France. It is composed by six nodes with loads, generators and a battery. Due to communication constraints, only a local description of the zone is available and the connection with the remaining network is defined as a perturbation acting on the zone.

In the sequel, we introduce two models describing the power and energy dynamics in the selected zone. The first model considers both the available renewable power and the actually produced one, but has nonlinear constraints. It receives information only about the available power variation. The second model considers the generated renewable power as state variable, and assumes availability of information on both available power and its variation. This results in a linear system with only linear constraints, but additional off-line data treatment is needed. The actuation delays in power variation are considered as follows: the generators react with a delay denoted $\tau>0$, while the battery has a delay $d>0$. We make the following hypothesis:

1) each generator produces the maximum available renewable power or the maximum allowed one;

2) only a higher level controller can reduce the power curtailment set-points. For this reason, the proposed controller deals only with curtailment increase;

3) due to the considered high voltage, losses due to the battery charge and discharge can be neglected;

4) the loads are constant.

\section{A. Modeling with exact limitation}

Let us consider that the state variables are the power flows on the lines $F_{i j}$, the generated power $P_{n}^{P}$ and the available one $P_{n}^{A}$, the curtailed power $P_{n}^{C}$ and the battery power output $P_{n}^{B}$, respectively, and the energy of the batteries $E_{n}^{B}$. The control inputs are the power variations of $P_{n}^{B}, P_{n}^{P}$ and $P_{n}^{C}$, i.e. $\Delta P_{n}^{B}, \Delta P_{n}^{P}$ and $\Delta P_{n}^{C}$, respectively, while the power variation $\Delta P_{n}^{A}$ of the available power is a disturbance acting on the system that is communicated to the TSO, and $\Delta P_{n}^{T}$ describes the unknown power variation disturbance due to power flow outside the considered zone. The parameter $T$ describes the sampling time. The dynamic equations are

$$
\left\{\begin{aligned}
F_{i j}(k+1)= & F_{i j}(k)+\sum_{n \in \mathcal{Z}^{B}} b_{i j}^{n} \Delta P_{n}^{B}(k-d) \\
& +\sum_{n \in \mathcal{Z}^{C}} b_{i j}^{n} \Delta P_{n}^{P}(k) \\
& +\sum_{n \in \mathcal{Z}^{N}} b_{i j}^{n} \Delta P_{n}^{T}(k), \forall(i j) \in \mathcal{Z}^{L} \\
P_{n}^{C}(k+1)= & P_{n}^{C}(k)+\Delta P_{n}^{C}(k-\tau), \forall n \in \mathcal{Z}^{C} \\
P_{n}^{B}(k+1)= & P_{n}^{B}(k)+\Delta P_{n}^{B}(k-d), \forall n \in \mathcal{Z}^{B} \\
E_{n}^{B}(k+1)= & E_{n}^{B}(k)-T c_{n}^{B}\left[P_{n}^{B}(k)+\Delta P_{n}^{B}(k-d)\right], \\
& \forall n \in \mathcal{Z}^{B} \\
P_{n}^{A}(k+1)= & P_{n}^{A}(k)+\Delta P_{n}^{A}(k), \forall n \in \mathcal{Z}^{C}
\end{aligned}\right.
$$

We denote $\bar{P}_{n}^{P}>0, \forall n \in \mathcal{Z}^{C}$ as the maximum power that can be produced by the $n$ renewable generator.The produced 
power is defined as either the available one or the maximum allowed by the (partial) curtailment:

$$
P_{n}^{P}(k)=\min \left(P_{n}^{A}(k), \bar{P}_{n}^{P}-P_{n}^{C}(k)\right) \forall k, \forall n \in \mathcal{Z}^{C} .
$$

Consequently, the produced power variation depends on the current power produced, the curtailed power and the available one. By defining the current power generation limit after curtailment:

$$
f_{n}^{P}(k)=\bar{P}_{n}^{P}-P_{n}^{C}(k)-\Delta P_{n}^{C}(k-\tau),
$$

one can define the effective available power for production:

$$
g_{n}^{P}(k)=\min \left(P_{n}^{A}(k)+\Delta P_{n}^{A}(k), f_{P}(k)\right),
$$

and consequently obtain the update in production:

$$
\Delta P_{n}^{P}(k)=g_{n}^{P}(k)-P_{n}^{P}(k) .
$$

We distinguish two disturbances. The values of $\Delta P_{n}^{A}(k)$ are communicated at each sampling $k$; consequently, $\Delta P_{n}^{P}(k)$ is known while its evolution over the next samplings is not. On the contrary, the values of the disturbance $\Delta P_{n}^{T}(k)$ are not known.

To describe the model in a compact form, we define:

$$
\begin{aligned}
F & =\operatorname{col}\left[F_{i j}\right], \forall(i, j) \in \mathcal{Z}^{L} ; \\
P^{C} & =\operatorname{col}\left[P_{n}^{C}\right], \Delta P^{C}=\operatorname{col}\left[\Delta P_{n}^{C}\right], \forall n \in \mathcal{Z}^{C} ; \\
P^{B} & =\operatorname{col}\left[P_{n}^{B}\right], \forall n \in \mathcal{Z}^{B} ; \\
E^{B} & =\operatorname{col}\left[E_{n}^{B}\right], \Delta P^{B}=\operatorname{col}\left[\Delta P_{n}^{B}\right], \forall n \in \mathcal{Z}^{B} ; \\
P^{A} & =\operatorname{col}\left[P_{n}^{A}\right], \Delta P^{A}=\operatorname{col}\left[\Delta P_{n}^{A}\right], \forall n \in \mathcal{Z}^{C} ; \\
\Delta P^{T} & =\operatorname{col}\left[\Delta P_{n}^{T}\right], \forall n \in \mathcal{Z}^{N} ; \\
P^{P} & =\operatorname{col}\left[P_{n}^{P}\right], \Delta P^{P}=\operatorname{col}\left[\bar{P}_{n}^{P}\right], \forall n \in \mathcal{Z}^{C}
\end{aligned}
$$

To correctly model the system dynamics, we define the upper and lower bounds of each variable as constant: $L_{i j}>0, \bar{P}_{n}^{C}>$ $0, \overline{\Delta P}_{n}^{C}>0 \bar{P}_{n}^{B}>0, \underline{P}_{n}^{B}<0, \bar{E}_{n}^{B}>0, \underline{E}_{n}^{B}>0, \underline{\Delta P}_{n}^{P}<0$, $\underline{\underline{\Delta P}}_{n}^{B}<0, \underline{\Delta P}^{A}{ }^{A}<0, \underline{\Delta P}_{n}^{T}<0, \overline{\Delta P}_{n}{ }^{T}>0, \overline{\Delta P}_{n}^{B}>0$, $\overline{\overline{\Delta P}}_{n}^{A}>0, \overline{\Delta P}_{n}^{T}>0$. Then,

$\bar{L}=\operatorname{col}\left[L_{i j}\right], \forall(i, j) \in \mathcal{Z}^{L}$;

$\underline{P}^{B}=\operatorname{col}\left[\underline{P}_{n}^{B}\right], \bar{P}^{B}=\operatorname{col}\left[\bar{P}_{n}^{B}\right], \forall n \in \mathcal{Z}^{B} ;$

$\bar{P}^{C}=\operatorname{col}\left[\bar{P}_{n}^{C}\right], \overline{\Delta P}^{C}=\operatorname{col}\left[\overline{\Delta P}_{n}^{C}\right], \forall n \in \mathcal{Z}^{C} ;$

$\underline{E}^{B}=\operatorname{col}\left[\underline{E}_{n}^{B}\right], \bar{E}^{B}=\operatorname{col}\left[\bar{E}_{n}^{B}\right], \forall n \in \mathcal{Z}^{B} ;$

$\underline{\Delta P}^{B}=\operatorname{col}\left[\underline{\Delta P}_{n}^{B}\right], \overline{\Delta P}^{B}=\operatorname{col}\left[\overline{\Delta P}_{n}^{B}\right], \forall n \in \mathcal{Z}^{B} ;$

$\overline{\Delta P}^{A}=\operatorname{col}\left[\Delta \bar{P}_{n}^{A}\right], \underline{\Delta P}^{A}=\operatorname{col}\left[\underline{\Delta P}_{n}^{A}\right], \forall n \in \mathcal{Z}^{C} ;$

$\overline{\Delta P}^{T}=\operatorname{col}\left[\Delta \bar{P}_{n}^{T}\right], \underline{\Delta P^{T}}=\operatorname{col}\left[\underline{\Delta P}_{n}^{T}\right], \forall n \in \mathcal{Z}^{C} ;$

$\bar{P}^{P}=\operatorname{col}\left[\bar{P}_{n}^{P}\right], \forall n \in \mathcal{Z}^{C}$;

$\underline{\Delta P}^{P}=\operatorname{col}\left[\underline{\Delta P}_{n}^{P}\right], \overline{\Delta P}^{P}=\operatorname{col}\left[\overline{\Delta P}_{n}^{P}\right], \forall n \in \mathcal{Z}^{C}$.
Consequently, supposing the disturbances to be bounded, the constraints are:

$$
\begin{aligned}
& -\bar{L} \leq F(k) \leq \bar{L}, \quad \mathbb{0}_{\mathbf{n}} \mathbf{C}_{\times \mathbf{1}} \leq P^{C}(k) \leq \bar{P}^{P}, \\
& \underline{P}^{B} \leq P^{B}(k) \leq \bar{P}^{B}, \quad \underline{E}^{B} \leq E^{B}(k) \leq \bar{E}^{B}, \\
& \mathbb{0}_{\mathbf{n}} \mathbf{C} \times \mathbf{1} \leq P^{A}(k) \leq \bar{P}^{P}, \quad \mathbb{0}_{\mathbf{n}} \mathbf{C}_{\times \mathbf{1}} \leq P^{P}(k) \leq \bar{P}^{P}, \\
& \mathbb{0}_{\mathbf{n}} \mathbf{C}_{\times \mathbf{1}} \leq \Delta P^{C}(k) \leq \overline{\Delta P}^{C}, \quad \underline{\Delta P}^{B} \leq \Delta P^{B}(k) \leq \overline{\Delta P}^{B},
\end{aligned}
$$

$$
\underline{\Delta P}^{T} \leq \Delta P^{T}(k) \leq \overline{\Delta P}^{T}, \quad \underline{P}^{A} \leq \Delta P^{A}(k) \leq \overline{\Delta P}^{A} .
$$

With respect to the description of the power coming from the renewables, the here proposed model describes the full set of variables to be considered as state variables, and considers only the external set of variables $\Delta P^{A}$ as known disturbances. However, it has several nonlinear constraints, due to the necessity to use min conditions to define the produced power that depends on $\Delta P^{C}(k-\tau)$. Due to the complexity to handle such constraints in an optimal control framework, in the sequel we target to modify the model in (2), (3) and (6) into a linear one with linear constraints.

\section{B. Modeling with approximate limitation}

Let us focus from a different angle to the previous model. Even if the $P_{n}^{P}(k)$ is not part of the state-space model, by exploiting (3) and (6), a nonlinear equation governing its evolution can be devised as

$$
P_{n}^{P}(k+1)=P_{n}^{P}(k)+\Delta P_{n}^{G}(k)-\Delta P_{n}^{C}(k-\tau)
$$

where, we aim to express in an explicit affine form the control action $\Delta P_{n}^{C}$ related to the curtailment and isolate the update in the generation $\Delta P_{n}^{G}(k)$ which can eventually be treated as an uncertainty. In particular, this term concentrating the uncertainty, $\Delta P_{n}^{G}(k)$, is defined as

$$
\Delta P_{n}^{G}(k)=\min \left(f_{n}^{G}(k), g_{n}^{G}(k)\right),
$$

with

$$
\begin{aligned}
& f_{n}^{G}(k)=P_{n}^{A}(k)+\Delta P_{n}^{A}(k)-P_{n}^{G}(k)+\Delta \hat{P}_{n}^{C}(k-\tau), \\
& g_{n}^{G}(k)=\bar{P}_{n}^{G}-P_{n}^{C}(k)-P_{n}^{G}(k) .
\end{aligned}
$$

To avoid confusion with the previous modeling in (2), we denote in the following the generated power by $P_{n}^{G}$ instead of the notation used previously for the produced one. Through this simple change of notation, the evolution of the production $P_{n}^{G}(k)$ follows:

$$
P_{n}^{G}(k+1)=P_{n}^{G}(k)+\Delta P_{n}^{G}(k)-\Delta P_{n}^{C}(k-\tau)
$$

and a linear dynamics is obtained based on the uncertain input signal $\Delta P_{n}^{G}(k)$ and the delayed control $\Delta P_{n}^{C}(k-\tau)$.

Globally, the state variables of the energy transmission are: the power flows on the lines $F_{i j}$, the generated power $P_{n}^{G}$, the curtailed power $P_{n}^{C}$ and the battery power output $P_{n}^{B}$, respectively, and the energy of the batteries $E_{n}^{B}$. The control inputs are the power variations $\Delta P_{n}^{B}$ and $\Delta P_{n}^{C}$. Finally, the 
power variation $\Delta P_{n}^{G}$ of the generated power $P_{n}^{G}$ is a filtered disturbance acting on the system. It is known at instant $k$ based on the state, control input and context information within the zone (available power). Indeed, the available power $P_{n}^{A}$ will not be part of the state vector. We suppose it is communicated to the TSO at each sampling time, together with the power variation $\Delta P_{n}^{A}$. Consequently, the value of $\Delta P_{n}^{G}$ is implicitly defined with respect to the communicated values of $P_{n}^{A}, \Delta P_{n}^{A}$, and the stored value of $P_{n}^{G}$ with respect to $P_{n}^{C}$.

The disturbance $\Delta P_{n}^{T}$ is unknown as in model (2) as it involves the information outside the operated zone.

With all these elements we can state the dynamical model:

$$
\left\{\begin{aligned}
F_{i j}(k+1)= & F_{i j}(k)+\sum_{n \in \mathcal{Z}^{B}} b_{i j}^{n} \Delta P_{n}^{B}(k-d) \\
& +\sum_{n \in \mathcal{Z}^{C}} b_{i j}^{n}\left[\Delta P_{n}^{G}(k)-\Delta P_{n}^{C}(k-\tau)\right] \\
& +\sum_{n \in \mathcal{Z}^{N}} b_{i j}^{n} \Delta P_{n}^{T}(k), \forall(i j) \in \mathcal{Z}^{L} \\
P_{n}^{C}(k+1)= & P_{n}^{C}(k)+\Delta P_{n}^{C}(k-\tau), \forall n \in \mathcal{Z}^{C} \\
P_{n}^{B}(k+1)= & P_{n}^{B}(k)+\Delta P_{n}^{B}(k-d), \forall n \in \mathcal{Z}^{B} \\
E_{n}^{B}(k+1)= & E_{n}^{B}(k)-T c_{n}^{B}\left[P_{n}^{B}(k)+\Delta P_{n}^{B}(k-d)\right], \\
& \forall n \in \mathcal{Z}^{B} \\
P_{n}^{G}(k+1)= & P_{n}^{G}(k)+\Delta P_{n}^{G}(k)-\Delta P_{n}^{C}(k-\tau), \\
& \forall n \in \mathcal{Z}^{C}
\end{aligned}\right.
$$

Philosophically, the difference in between equation (3) and (10) with the proposed simplification is similar to the one in between a dynamical system with nonlinearity in control and its linearized counterpart, i.e.

$$
\begin{aligned}
x(k+1) & =x(k)+f(x(k), u(k), w(k)) \\
& =x(k)+\underbrace{g(x(k), u(k), w(k))}_{v(k)}+u(k) \\
& =x(k)+v(k)+u(k)
\end{aligned}
$$

The main advantage of the proposed modeling concerns the possibility to pre-compute the term $\Delta P_{n}^{G}(k)$, based on values of $P^{A}(k), P^{G}(k), P^{C}(k), \Delta P^{A}(k)$ and $\Delta P^{C}(k-\tau)$, while maintaining the system linear via the offline computation of the min and consequently reducing the computational effort of dedicated model-based predictive control laws. The main drawback is related to the term $\Delta P^{C}(k-\tau)$. Whenever this is an independent variable at the pre-computation time of sampling instant $k$, then the model $(15)$ is equivalent to model (2) as long as the first one is implicitly nonlinear.

To avoid this implicit dependence in (15), a predictioncorrection mechanism can be used, in particular for control design. Indeed, one can purposely consider a predicted value, e.g. $\Delta \hat{P}_{n}^{C}(k-\tau)=0$, in (12) and dissociate it from the actual selection of $\Delta P_{n}^{C}(k-\tau)$, which is the control input to be fixed at time $k$. The linearity of the prediction model is preserved and its evolution can be corrected once $\Delta P_{n}^{C}(k-\tau)$ is chosen. This mechanism implies a model mismatch in between the prediction and the correction phase, whenever $\Delta P_{n}^{C}(k-\tau) \neq \Delta \hat{P}_{n}^{C}(k-\tau)$. However, since the curtailement action is not supposed to take place frequently, and since at each sampling time a new state measurement is available (correction), the model mismatch is aimed not to impact significantly the precision all by preserving a linear structure of the mathematical model.

Following the notation in (16), for the prediction purpose, the following equation is employed:

$$
\hat{x}(k+1)=x(k)+\hat{v}(k)+u(k)
$$

where the value of the disturbance $\hat{v}$ is predicted (with appropriate assumptions) in order to obtain the optimal control input $u^{*}(k)=u^{*}(x(k), \hat{v})$. Subsequently, the update equation which accounts for the optimal control value based on the production will be:

$$
\begin{aligned}
x(k+1) & =x(k)+v(k)+u^{*}(x(k), \hat{v}) \\
& =x(k)+v\left(u^{*}(x(k), \hat{v})\right)+u^{*}(x(k), \hat{v})
\end{aligned}
$$

Then, the difference between the linearised case and the nonlinear one relies in the implementation of equation (11). When the value $\Delta P_{n}^{C}(k-\tau)$ is not known, then the implicit nonlinear system is linearised by using the prediction-correction with respect to $\Delta \hat{P}_{n}^{C}(k-\tau)$.

Using the same formalism of (7a)-(7f), we define

$$
\begin{aligned}
& P^{G}=\operatorname{col}\left[P_{n}^{G}\right], \quad \Delta P^{G}=\operatorname{col}\left[\Delta P_{n}^{G}\right], \forall n \in \mathcal{Z}^{C} ; \\
& \bar{P}^{G}=\operatorname{col}\left[\bar{P}_{n}^{G}\right], \forall n \in \mathcal{Z}^{C} ;
\end{aligned}
$$

where $\bar{P}_{n}^{G}>0$ is the maximum power that can be generated by the $n$ renewable power plant, with $n \in \mathcal{Z}^{C}$. Then:

$$
\begin{aligned}
& -\bar{L} \leq F(k) \leq \bar{L}, \quad \mathbb{0}_{\mathbf{n}^{\mathbf{C}} \times \mathbf{1}} \leq P^{C}(k) \leq \bar{P}^{G}, \\
& \underline{P}^{B} \leq P^{B}(k) \leq \bar{P}^{B}, \quad \underline{E}^{B} \leq E^{B}(k) \leq \bar{E}^{B}, \\
& \mathbb{O}_{\mathbf{n}^{\mathbf{C}} \times \mathbf{1}} \leq P^{G}(k) \leq \bar{P}^{G} .
\end{aligned}
$$

Furthermore, the following constraints apply:

$$
\begin{gathered}
P_{n}^{G}(k) \leq \bar{P}_{n}^{G}-P_{n}^{C}(k), \forall n \in \mathcal{Z}^{C} . \\
P_{n}^{G}(k) \leq P_{n}^{A}(k), \forall k, \forall n \in \mathcal{Z}^{C} .
\end{gathered}
$$

Consequently, bounds for $\Delta P_{n}^{G}(k)$ must be coherent with respect to the available power variation $\Delta P_{n}^{A}(k)$ and the maximum allowed power $\bar{P}_{n}^{G}-P_{n}^{C}(k)$.

According to (7a)-(7f) and 22a), the resulting linear system is described as

$$
\begin{aligned}
x(k+1)= & A x(k)+B_{C} u_{C}(k-\tau)+B_{B} u_{B}(k-d) \\
& +D_{w} w(k)+D_{\zeta} \zeta(k)
\end{aligned}
$$

where

$$
\begin{aligned}
& x(k)=\left[\begin{array}{lllll}
F(k) & P^{C}(k) & P^{B}(k) & E^{B}(k) & P^{G}(k)
\end{array}\right]^{T}, \\
& u_{C}(k)=\Delta P^{C}(k), \quad u_{B}(k)=\Delta P^{B}(k), \\
& w(k)=\Delta P^{G}(k), \quad \zeta(k)=\Delta P^{T}(k),
\end{aligned}
$$


$A=\left(\begin{array}{ccccc}\mathbb{1}_{\mathbf{n}^{\mathrm{L}} \times \mathbf{n}^{\mathrm{L}}} & \mathbb{O}_{\mathbf{n}^{\mathrm{L}} \times \mathbf{n}^{\mathrm{C}}} & \mathbb{O}_{\mathbf{n}^{\mathrm{L}} \times \mathbf{n}^{\mathrm{B}}} & \mathbb{O}_{\mathbf{n}^{\mathrm{L}} \times \mathbf{n}^{\mathrm{B}}} & \mathbb{O}_{\mathbf{n}^{\mathrm{L}} \times \mathbf{n}^{\mathrm{C}}} \\ \mathbb{O}_{\mathbf{n}^{\mathbf{C}} \times \mathbf{n}^{\mathrm{L}}} & \mathbb{1}_{\mathbf{n}^{\mathrm{C}} \times \mathbf{n}^{\mathrm{C}}} & \mathbb{O}_{\mathbf{n}^{\mathrm{C}} \times \mathbf{n}^{\mathrm{B}}} & \mathbb{O}_{\mathbf{n}} \mathbf{n}^{\mathrm{C}} \times \mathbf{n}^{\mathrm{B}} & \mathbb{O}_{\mathbf{n}^{\mathrm{C}} \times \mathbf{n}^{\mathrm{C}}} \\ \mathbb{O}_{\mathbf{n}^{\mathrm{B}} \times \mathbf{n}^{\mathrm{L}}} & \mathbb{O}_{\mathbf{n}^{\mathrm{B}} \times \mathbf{n}^{\mathrm{C}}} & \mathbb{1}_{\mathbf{n}^{\mathrm{B}} \times \mathbf{n}^{\mathrm{B}}} & \mathbb{O}_{\mathbf{n}^{\mathrm{B}} \times \mathbf{n}^{\mathrm{B}}} & \mathbb{O}_{\mathbf{n}^{\mathrm{B}} \times \mathbf{n}^{\mathrm{C}}} \\ \mathbb{O}_{\mathbf{n}^{\mathrm{B}} \times \mathbf{n}^{\mathrm{L}}} & \mathbb{O}_{\mathbf{n}^{\mathrm{B}} \times \mathbf{n}^{\mathrm{C}}} & -A_{b} & \mathbb{1}_{\mathbf{n}^{\mathrm{B}} \times \mathbf{n}^{\mathrm{B}}} & \mathbb{O}_{\mathbf{n}^{\mathrm{B}} \times \mathbf{n}^{\mathrm{C}}} \\ \mathbb{O}_{\mathbf{n}^{\mathrm{C}} \times \mathbf{n}^{\mathrm{L}}} & \mathbb{O}_{\mathbf{n}^{\mathrm{C}} \times \mathbf{n}^{\mathrm{C}}} & \mathbb{O}_{\mathbf{n}^{\mathrm{C}} \times \mathbf{n}^{\mathrm{B}}} & \mathbb{O}_{\mathbf{n}^{\mathbf{C}} \times \mathbf{n}^{\mathrm{B}}} & \mathbb{1}_{\mathbf{n}^{\mathrm{C}} \times \mathbf{n}^{\mathrm{C}}}\end{array}\right)$,

$$
B_{C}=\left(\begin{array}{c}
-M_{c} \\
\mathbb{1}_{\mathbf{n}^{\mathbf{C}} \times \mathbf{n}^{\mathbf{C}}} \\
\mathbb{O}_{\mathbf{n}^{\mathrm{B}} \times \mathbf{n}^{\mathbf{C}}} \\
\mathbb{O}_{\mathbf{n}^{\mathrm{B}} \times \mathbf{n}^{\mathbf{C}}} \\
-\mathbb{1}_{\mathbf{n}^{\mathbf{C}} \times \mathbf{n}^{\mathbf{C}}}
\end{array}\right), \quad B_{B}=\left(\begin{array}{c}
M_{b} \\
\mathbb{O}_{\mathbf{n}^{\mathbf{C}} \times \mathbf{n}^{\mathrm{B}}} \\
\mathbb{1}_{\mathbf{n}^{\mathrm{B}} \times \mathbf{n}^{\mathrm{B}}}-A_{b} \\
\mathbb{O}_{\mathbf{n}^{\mathbf{C}} \times \mathbf{n}^{\mathrm{B}}}
\end{array}\right),
$$

$$
D_{w}=\left(\begin{array}{ccccc}
M_{c} & \mathbb{O}_{\mathbf{n}^{\mathbf{C}} \times \mathbf{n}^{\mathbf{C}}} & \mathbb{O}_{\mathbf{n}^{\mathrm{B}} \times \mathbf{n}^{\mathbf{C}}} & \mathbb{O}_{\mathbf{n}^{\mathrm{B}} \times \mathbf{n}^{\mathbf{C}}} & \mathbb{1}_{\mathbf{n}^{\mathbf{C}} \times \mathbf{n}^{\mathbf{C}}}
\end{array}\right)^{T},
$$$$
D_{\zeta}=\left(\begin{array}{lllll}
M_{t} & \mathbb{O}_{\mathbf{n}^{\mathbf{C}} \times \mathbf{n}^{\mathrm{N}}} & \mathbb{O}_{\mathbf{n}^{\mathrm{B}} \times \mathbf{n}^{\mathrm{N}}} & \mathbb{O}_{\mathbf{n}^{\mathrm{B}} \times \mathbf{n}^{\mathrm{N}}} & \mathbb{O}_{\mathbf{n}} \mathbf{C}_{\times \mathbf{n}^{\mathrm{N}}}
\end{array}\right)^{(32},
$$

with $A_{b}=\operatorname{diag}\left[T c_{n}^{B}\right], \forall n \in \mathcal{Z}^{B}$, and $M_{c}, M_{b}$ and $M_{t}$ that are composed by the elements $b_{i j}^{n}$ of the PTDF matrix described in 15. The $k^{t h}$ line in these matrices corresponds to the PTDF of the $k^{t h}$ line of $F_{i j}$ at nodes where generation can be curtailed, at nodes where a battery is installed or at nodes where the injections may vary, respectively.

\section{Simulations}

In this Section, we show a comparison among the proposed linear modeling in 15 based on the simulation function of MATPOWER [10], i.e. runpf. The function runpf runs a power flow based on an AC modeling, which seems compliant with the $5 \mathrm{~s}$ sampling time we consider. A first target of the present paper is to provide a control-oriented dynamical system that performs similarly to runpf. We remark that the proposed model describes only a small zone (e.g. 6 busses), while the functions runpf take into account the whole transmission network ( $\sim 6000$ busses). For sake of simplicity (and without loss of generality as long as the simulation is performed in open-loop) in the simulation scenario we consider $\tau=d=0$.

In the sequel, the simulation based on runpf is deemed relevant for the network behaviour, and a comparison is made with respect to a one-step ahead prediction based on the measured value that is taken at each sampling or twenty-step ahead prediction. Since at minimum prediction horizon of 50 seconds must be considered for control purposes, such choice of the measure updates is consistent with the needed time to curtail the generated power, i.e. $\tau=9$ since the delay is 45 seconds. Two situations are analysed:

a) a comparison between runpf, and the system in (15) with $\Delta \hat{P}_{n}^{C}(k-\tau)=\Delta P_{n}^{C}(k-\tau)$

b) a comparison between runpf, and the system in 15 with $\Delta \hat{P}_{n}^{C}(k-\tau)=0$.

We consider the b) case in order to show the difference between the real system and the simplified one. The considered zone is composed by six nodes, where four of them have renewable power generators, and one has a battery. The considered power generators have a maximum installed power of $78 \mathrm{MW}$, while the battery is a $10 \mathrm{MW}$ one. We consider real data for wind turbine power production.

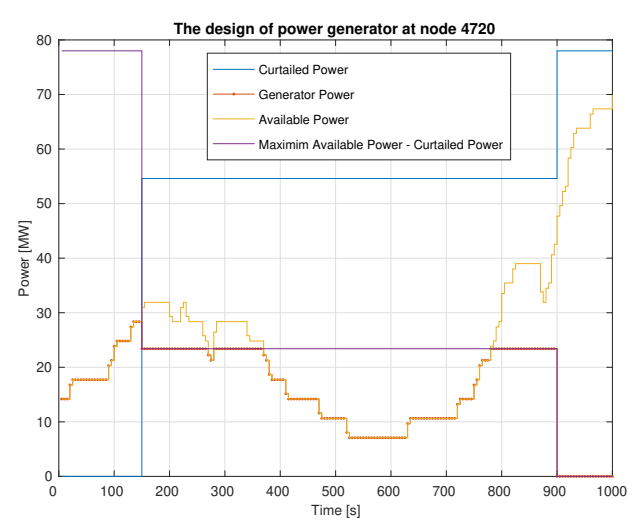

Fig. 2. The power variables in the power generator at node 4720. The $P_{n}^{C}(k)$ variable is described by the blue curve, while the violet one depicts $\bar{P}_{n}^{G}-$ $P_{n}^{C}(k)$. The yellow line represents $P_{n}^{A}(k)$, and the dotted red one is $P_{n}^{G^{n}}(k)$.

Figure 2 depicts the power variables with respect to the generator in node 4720 . We consider a given curtailed power profile, such that the allowed maximum power is:

- $100 \%$ between 0 and 150 seconds;

- $30 \%$ between 150 and 900 seconds;

- $0 \%$ after 900 seconds.

The $P_{n}^{C}(k)$ variable is described by the blue curve, while the violet one depicts $\bar{P}_{n}^{G}-P_{n}^{C}(k)$. The yellow line represents $P_{n}^{A}(k)$, and the dotted red one is $P_{n}^{G}(k)$. The power profile for $P_{n}^{A}(k)$ is taken by interpolation of real data that are measured each 10 seconds. Figure 2 confirms that the modeling proposed in equation 15 correctly manages to describe the different situations it can faces. As described in Section II-B, the simulation is carried on under the the hypothesis of a known and estimated value of $\Delta P_{n}^{C}(k-\tau)$. Consequently, different results due to the cases a) and b) in Figure 3 and 4 respectively are presented. We focus only on one branch for sake of space.

Figures 3 and 4 depict the power along the line between nodes 4720 and 1445 . Due to DC nature of the proposed modeling, we remark that the proposed model matches the AC one computed by runpf with a small mismatch when $P_{n}^{C}(k)=P_{n}^{C}(k+1)$. As expected, the proposed model is closer to the emulated one when the state is measured each sampling, both in the case a) (Figure 3) and in case b) (Figure 4). Differences in the results are expected when $\Delta P_{n}^{C}(k) \neq 0$, i.e. at time $150 \mathrm{~s}$ and $900 \mathrm{~s}$. Indeed, the modeling error $\Delta P_{n}^{C}(k)=0$ in the simplified model generates a prediction error, as shown at time $150 \mathrm{~s}$ in Figure 4. The estimation mismatch of $\Delta \hat{P}_{n}^{C}(k)$ generates a prediction error that is similar in both cases A and B in Figure 4 at time 150s: however, as A is updated at each sample, its evolution is corrected to the real value at time $155 \mathrm{~s}$, while it happens at time $200 \mathrm{~s}$ for B. On the contrary, no mismatch due to the wrong value of $\Delta \hat{P}_{n}^{C}(k)$ take place at time $900 \mathrm{~s}$. This is due to the fact that the value of equation (11) is defined by $g_{n}^{G}$ in (13), and consequently there is no modeling error. We remark then that the adopted simplification generates errors in a subset of the possible cases, and depends on the combination of the 


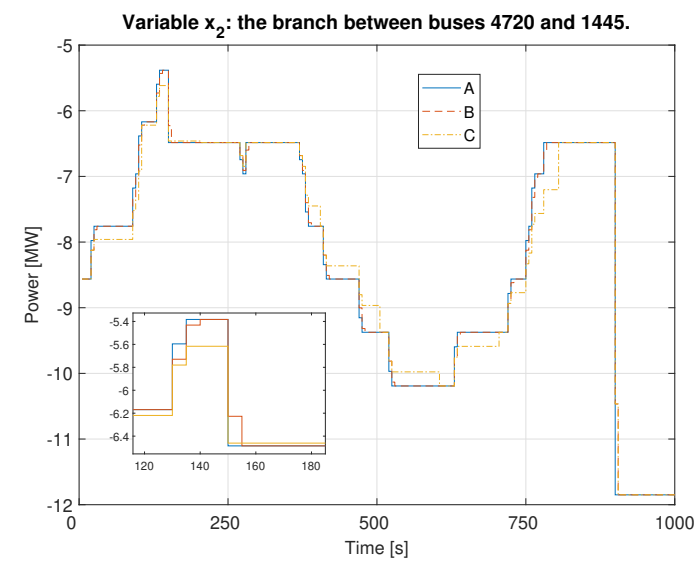

Fig. 3. Case a): The power flow on the branch between buses 4720 and 1445 . A) Runpf. B) System updated each sampling. C) System updated every 20 samplings.

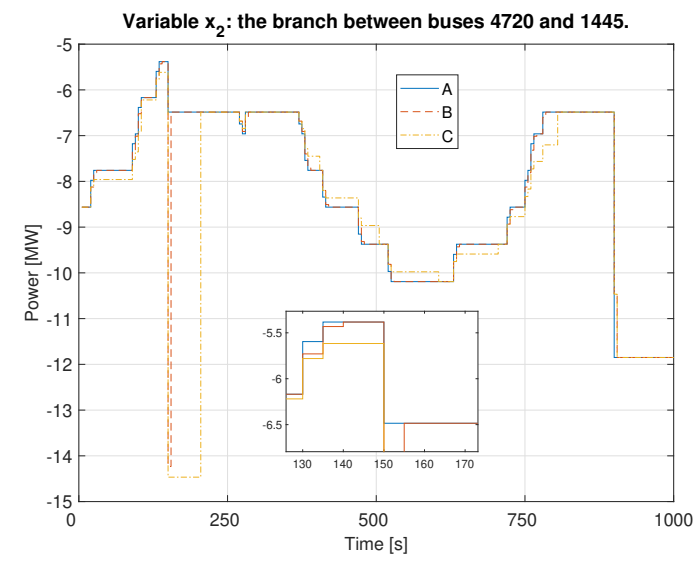

Fig. 4. Case b): The power flow on the branch between buses 2745 and 10000 . A) Runpf. B) System updated each sampling. C) System updated every 20 samplings.

current values of $P_{n}^{A}(k)$ and $P_{n}^{C}(k)$. The proposed model can then be used to describe the AC transmission power network. As expected, higher errors correspond to slowest updates, both when considering the nonlinear model or the simplified linear one. However, the difference between the predicted dynamics and the real one is reasonably close almost everywhere, and the possibility to measure the real state contributes on reducing the model mismatch. Then, the capability of the proposed model to describe the real system is verified, and it can be used for model based control approaches.

\section{CONCLUSiOnS}

A control-oriented dynamical system is proposed to model the power variation taking place in a subtransmission area. The necessity to model the possibility to partially curtail the renewable power requests the consideration of a richer model with respect to the ones usually investigated. The proposed solution uses PTDF and results to be a linear model with linear constraints. A comparison with the close to reality simulation tool MATPOWER is provided, and the linear model describing only a zone is shown to produce satisfactory results with respect to the ones obtained by the whole real system emulation. Future work will consider dedicated control methods to optimally address the target to manage the power flow in the considered zone.

\section{ACKNOWLEDGEMENT}

This work was carried out within the CPS4EU project, which has received funding from the ECSEL Joint Undertaking (JU) under grant agreement No 826276. The JU receives support from the European Union's Horizon 2020 research and innovation programme and France, Spain, Hungary, Italy, Germany. The proposed results reflect only the authors' view. The JU is not responsible for any use that may be made of the information the present work contains.

\section{REFERENCES}

[1] B. Meyer, J. Astic, P. Meyer, F. Sardou, C. Poumarede, N. Couturier, M. Fontaine, C. Lemaitre, J. Maeght, and C. Straub, "Power Transmission Technologies and Solutions: The Latest Advances at RTE, the French Transmission System Operator," IEEE Power and Energy Magazine, vol. 18, no. 2, pp. 43-52, 2020.

[2] C. Straub, S. Olaru, J. Maeght, and P. Panciatici, "Zonal congestion management mixing large battery storage systems and generation curtailment," in 2018 IEEE Conference on Control Technology and Applications (CCTA), pp. 988-995, 2018.

[3] C. Straub, S. Olaru, J. Maeght, and P. Panciatici, "Robust MPC for temperature management on electrical transmission lines," vol. 51, pp. 355 - 360, 2018. 17th IFAC Workshop on Control Applications of Optimization CAO 2018.

[4] M. Almassalkhi and I. Hiskens, "Temperature-based model-predictive cascade mitigation in electric power systems," in 52nd IEEE Conference on Decision and Control, pp. 7560-7567, 2013.

[5] M. R. Almassalkhi and I. A. Hiskens, "Model-predictive cascade mitigation in electric power systems with storage and renewables-part i: Theory and implementation," IEEE Transactions on Power Systems, vol. 30, no. 1, pp. 67-77, 2015.

[6] M. R. Almassalkhi and I. A. Hiskens, "Model-predictive cascade mitigation in electric power systems with storage and renewables_part ii: Case-study," IEEE Transactions on Power Systems, vol. 30, no. 1, pp. 78-87, 2015.

[7] E. F. Camacho and C. Bordons, Model predictive control. Springer, 2007.

[8] A. J. Wood, B. F. Wollenberg, and G. B. Sheblé, Power Generation, Operation, and Control, 3rd Edition. Wiley, 2013.

[9] Xu Cheng and T. J. Overbye, "PTDF-based power system equivalents," IEEE Transactions on Power Systems, vol. 20, no. 4, pp. 1868-1876, 2005.

[10] R. D. Zimmerman, C. E. Murillo-Sánchez, and R. J. Thomas, "Matpower: Steady-state operations, planning, and analysis tools for power systems research and education," IEEE Transactions on Power Systems, vol. 26, no. 1, pp. 12-19, 2011. 